\title{
QED corrections to leptonic decay rates
}

\author{
P. A. Boyle ${ }^{2}$, V. Gülpers ${ }^{2}$, A. Jüttner ${ }^{1}$, C. Lehner ${ }^{3}$, F. Ó hÓgáin ${ }^{2}$, A. Portelli², \\ J. P. Richings ${ }^{* 1}$, C.T. Sachrajda ${ }^{1}$, \\ ${ }^{1}$ School of Physics and Astronomy, University of Southampton, Southampton SO17 1BJ, UK \\ ${ }^{2}$ School of Physics and Astronomy, University of Edinburgh, Edinburgh EH9 3JZ, UK \\ ${ }^{3}$ Physics Department, Brookhaven National Laboratory, Upton, NY 11973, USA \\ E-mail: j.p.richingsesoton.ac.uk
}

$\mathrm{RBC} / \mathrm{UKQCD}$ is preparing a calculation of leptonic decay rates including isospin breaking corrections using a perturbative approach to include NLO contributions from QED effects. We present preliminary numerical results for a contribution to the leptonic pion decay rate and report on exploratory studies of computational techniques based on all-to-all propagators.

The 36th Annual International Symposium on Lattice Field Theory - LATTICE2018

22-28 July, 2018

Michigan State University, East Lansing, Michigan, USA.

${ }^{*}$ Speaker. 


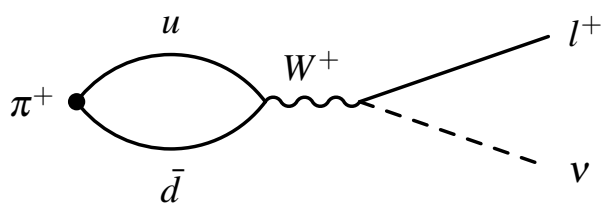

Figure 1: Pion decay into leptons via a weak current without QED contributions.

\section{Introduction}

In this contribution we outline RBC/UKQCD's work towards a Lattice QCD calculation of the isospin-breaking (IB) corrections to the leptonic decay rate for pions and kaons. This study is motivated by the sub-percent-level precision achieved by some collaborations for the calculation of $f_{\pi}$ and $f_{K}$, using various lattice actions in the isospin-symmetric limit, where up and down quarks are treated as identical particles [3]. The aim is to determine CKM-matrix elements from leptonic decays (Figure 1), thus enabling precise tests of the Standard Model. To date, one calculates decay constants on the lattice and then uses experimental results for the decay rates to yield the CKM matrix elements using

$$
\Gamma\left(\pi^{+} \rightarrow l^{+} v\right)=\frac{m_{\pi}}{8 \pi} G_{F}^{2}\left|f_{\pi^{+}}\right|^{2}\left|V_{u d}\right|^{2} m_{l}^{2}\left(1-\frac{m_{l}^{2}}{m_{\pi}^{2}}\right)^{2} .
$$

The pion decay constant $f_{\pi^{+}}$is defined in terms of the QCD matrix element, $\left\langle 0\left|\bar{d} \gamma_{\mu} \gamma_{5} u\right| \pi^{+}(p)\right\rangle=$ $i p_{\mu} f_{\pi^{+}}$, which is computed on the lattice from Euclidean two-point correlation functions. To further improve the precision isospin-breaking (IB) effects due to the different masses of the light quarks and the difference in the QED coupling between up- and down-type quarks must be taken into account. Based on power counting in the electromagnetic coupling and the up- and down-quark mass difference, respectively, one expects these effects to enter at the percent level.

We focus our discussion on the QED isospin-breaking corrections to leptonic decays of pions, following the approach developed in [1], where the QCD+QED path integral is expanded in $\alpha$ and IR divergences are dealt with consistently. Naively, QED in a finite volume is ill-defined due to the appearance of photon zero modes. We subtract these by hand within the framework of $\mathrm{QED}_{\mathrm{L}}[4,5,6,7]$. From amongst the various possible strategies for implementing the computation we here present one based on the use of all-to-all propagators [2]. In particular, we compute a number of low-mode eigenvectors exactly using a variant of the Lanczos algorithm [14]. The complement of the low-mode space is then estimated stochastically. As detailed below this setup allows to compute contractions of quark propagators (not only the ones required for the QED corrections) off-line, i.e., without requiring a super computer.

\section{QED Isospin Breaking corrections to leptonic decay rates}

In order to calculate the infra-red (IR) finite order- $\alpha$ leptonic decay rate, we must consider contributions from graphs with and without final state photons to cancel IR divergences [9]. Here we follow the strategy outlined in [1] to carefully deal with IR divergences, where the contributions with final state photons are treated analytically using the point-like approximation. The lattice 


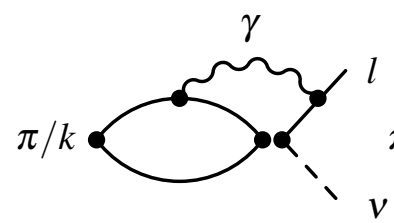

(a)

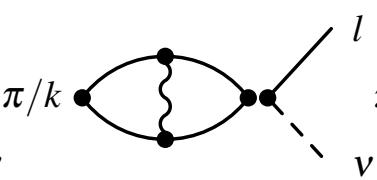

(b)

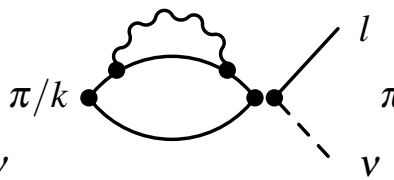

(c)

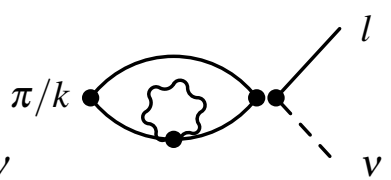

(d)

Figure 2: The four connected contributions to the QED-isospin-breaking correction to leptonic decay of a meson without final-state photon. The perturbative expansion contains the (a) lepton coupling, (b) exchange, (c) self-energy and (d) tadpole diagrams.

computation therefore only has to deal with diagrams without final-state photons for which the perturbative expansion of the QCD+QED path integral [10] to order $\alpha$ generates the set of diagrams illustrated in figure 2. We use Feynman gauge for which the photon propagator takes the form,

$$
\Delta_{\mu v}(x-y)=\delta_{\mu v} \frac{1}{L^{3} T} \sum_{k, \vec{k} \neq 0} \frac{e^{i k .(x-y)}}{\hat{k}^{2}}=\left\langle A_{\mu}(x) A_{v}(y)\right\rangle,
$$

where $\hat{k}=\frac{a}{2} \sin \left(\frac{a k}{2}\right)$ is the lattice momentum of the photon and $L$ and $T$ the spatial and time extent of the lattice, respectively. In practice, the photon propagator is generated by inserting stochastic photons [11]. Figure 2 shows illustrations of connected contributions without final-state photon. Figure $2 \mathrm{a}$ is a contribution where the photon couples to a quark and a lepton. We implement the lepton propagator on the lattice as a free domain wall fermion. As a first test the we calculated diagram in Figure 2a using sequential propagators [12] on a $24^{3} \times 64$ lattice. The ensemble used has an isospin symmetric pion mass of $340 \mathrm{MeV}$ and inverse lattice spacing of $a^{-1}=1.78 \mathrm{GeV}$ [13].

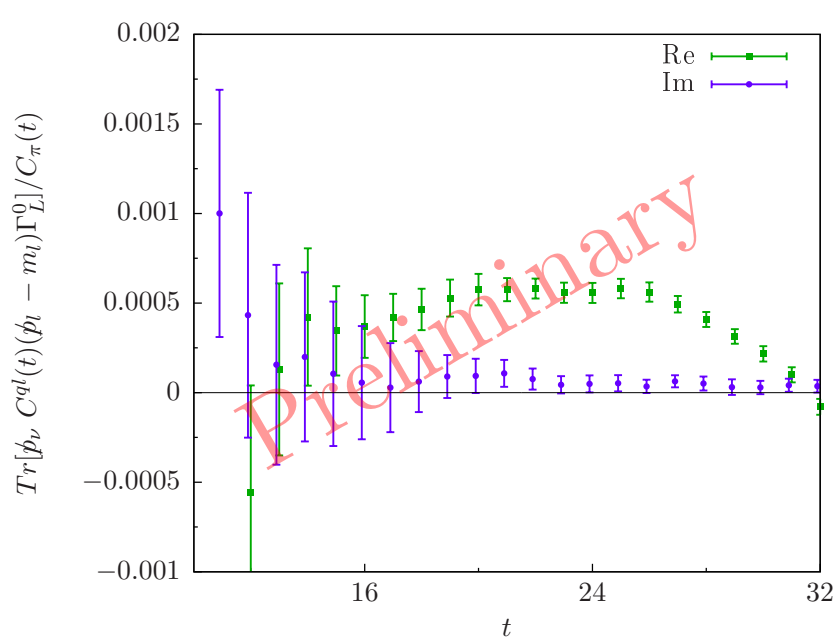

Figure 3: The plot shows the real (green) and imaginary (blue) components of the correlator for the diagram in figure 2a when the leptonic trace is formed. The horizontal axis is the time difference between the weak operator and the meson source positions. The vertical axis is the correlator for the lepton coupling diagram $C^{q l}$ with the trace over the free spin indices completed by including the factor $\left(\not p_{l}-m_{l}\right) \Gamma_{L}^{0} \not p_{v}$, normalised by the pion correlator.

The results for diagram 2a illustrated in Figure 3 shows an encouraging signal but we also investigate other methods which might offer better signal.

\section{The all-to-all approach and meson fields}

We follow the all-to-all approach in [2] where the propagator is decomposed into a number of exact 
low-mode eigenvectors the complement which is solved stochastically. We now describe how this offers a convenient way of structuring the calculation of correlators. Following [2] the all-to-all propagator can be constructed from two sets of vectors, $v_{i}(x)$ and $w_{i}(x)$, such that

$$
D_{\mathrm{A} 2 \mathrm{~A}}^{-1}(x, y)=\sum_{i=1}^{N_{\text {modes }}} v_{i}(x) w_{i}^{\dagger}(y),
$$

where $v_{l}(x)=\phi_{l}(x)$ and $w_{l}(y)=\phi_{l}(y) / \lambda_{l}$ are exact eigenvectors of the Dirac operator $\phi_{l}(x)$ with eigenvalues $\lambda_{l}$. In practice we only ever compute a limited number $N_{l}$. We then estimate their complement stochastically. In particular, we write

$$
D_{\mathrm{A} 2 \mathrm{~A}}^{-1}(x, y)=\sum_{l=1}^{N_{l}} v_{l}(x) w_{l}^{\dagger}(y)+\sum_{h=N_{l}+1}^{N_{\text {modes }}} v_{h}(x) w_{h}^{\dagger}(y),
$$

where $N_{\text {modes }}=N_{\text {high }}+N_{\text {low }}$. To calculate the high modes we use stochastic noise sources $\eta_{h}=$ $\{ \pm 1\}+i\{ \pm 1\}=w_{h}$ from which we project out the low mode contribution to the propagator,

$$
v_{h}(x)=\left(D^{-1}-\sum_{l=1}^{N_{l}} \phi_{l}(x) \phi_{l}^{\dagger}(x) / \lambda_{l}\right) \eta_{h}(x) .
$$

\subsection{Two point correlation function}

We can consider a two point correlation function and rewrite it in terms of all-to-all propagators using (3.1),

$$
\begin{aligned}
C_{\Gamma_{1} \Gamma_{2}}\left(t_{y}-t_{x}\right) & =\sum_{\vec{x}, \vec{y}} \operatorname{Tr}\left[\Gamma_{1} S(x, y) \Gamma_{2} S(y, x)\right]=\sum_{\vec{x}, \vec{y}} t r\left[\Gamma_{1} \sum_{i=1}^{N_{\text {modes }}} v_{i}(x) w_{i}^{\dagger}(y) \Gamma_{2} \sum_{j=1}^{N_{\text {modes }}} v_{j}(y) w_{j}^{\dagger}(x)\right] \\
& =\sum_{i, j=1}^{N_{\text {modes }}} \operatorname{tr}\left[\Pi_{i j}\left(t_{x} ; \Gamma\right) \Pi_{i j}\left(t_{y} ; \Gamma\right)\right],
\end{aligned}
$$

where we have introduced the shorthand

$$
\Pi_{i j}\left(t_{x} ; \Gamma\right)=\sum_{\vec{x}} w_{i}^{\dagger}(x) \Gamma v_{j}(x),
$$

which we refer to colloquially as meson field. Here, $\Gamma$ represents any choice of gamma matrix. Meson fields $\Pi_{i j}$ are of size $N_{T} \times N_{\text {modes }}^{2}$, where $N_{T}$ is the time extent of the lattice. The spatially summed meson fields can be stored to disk and retrieved later, i.e., off-line, to compute traces of products of it. By including a phase factor the meson fields can be projected on any desired lattice momentum. In this way we can construct, for instance, the two-point function between pseudoscalar and axial-vector currents,

$$
C_{P A}\left(t_{y}-t_{x}\right)=\sum_{i, j} \operatorname{tr}\left[\Pi_{i j}\left(t_{x} ; \gamma_{5}\right) \Pi_{j i}\left(t_{y} ; \gamma_{0} \gamma_{5}\right)\right]
$$

from which the lepton decay constant is determined. 


\section{Meson fields and isospin-breaking corrections to leptonic decays}

Here we discuss the construction of the meson fields that are required for computing the QEDisospin corrections to leptonic decay. In particular, we consider the quark-photon coupling via the conserved vector current.

\subsection{Point-split-operator meson fields}

For simplicity we consider the case of the conserved current for Wilson fermions,

$$
V_{\mu}^{c}(x)=\frac{1}{2}\left[\bar{\psi}(x+\hat{\mu})\left(1+\gamma_{\mu}\right) U_{\mu}^{\dagger}(x) \psi(x)-\bar{\psi}(x)\left(1-\gamma_{\mu}\right) U_{\mu}(x) \psi(x+\hat{\mu})\right] .
$$

The structure of Eq. (4.1) suggests that we require meson fields for operators with gauge-invariant point-split structure. The exchange diagram as illustrated in Fig. 2b, with photons inserted using conserved vector currents, has the form,

$$
C^{\text {exch. }}\left(t_{y}-t_{x}\right)=\sum_{\vec{x}, \vec{y}, r, s}\left\langle\bar{\psi}(y) \Gamma_{1} \psi(y) V_{\mu}^{c}\left(z_{1}\right) \bar{\psi}(x) \Gamma_{2} \psi(x) V_{v}^{c}\left(z_{2}\right) \Delta_{\mu v}(x-y)\right\rangle .
$$

We now rewrite the conserved-current contribution to this correlator in terms of a meson field. Concentrating on the second term in Eq. (4.1) we write

$$
\begin{aligned}
\sum_{z_{1}}\left\langle\ldots \Gamma_{1} \psi(y)[\right. & \left.\left.\bar{\psi}\left(z_{1}\right)\left(1-\gamma_{\mu}\right) U_{\mu}\left(z_{1}\right) \psi(y+\hat{\mu})\right] \bar{\psi}(x) \Gamma_{2} \ldots\right\rangle \\
& =\sum_{\vec{z}_{1}}\left\langle\ldots \Gamma_{1} S\left(y, z_{1}\right)\left(1-\gamma_{\mu}\right) U_{\mu}\left(z_{1}\right) S\left(z_{1}+\hat{\mu}, x\right) \Gamma_{2} \ldots\right\rangle,
\end{aligned}
$$

where we have carried out the Wick contractions in the 2nd line. Rewriting the propagators in terms of the all-to-all decomposition (3.1),

$$
\sum_{i j}\left\langle\ldots v_{i}(y)\left[\sum_{z_{1}} w_{i}^{\dagger}\left(z_{1}\right)\left(1-\gamma_{\mu}\right) U_{\mu}\left(z_{1}\right) v_{j}\left(z_{1}+\hat{\mu}\right)\right] w_{j}^{\dagger}(x) \ldots\right\rangle .
$$

The square brackets on the right of (4.4) contain a meson field. We can treat the first term in (4.1) in the same way and thereby obtain a meson field for the conserved vector current. Including a stochastic photon field $A_{\mu}(x)$ is straight-forward,

$\Pi_{i j}\left[t_{x}, V_{\mu}^{c} A_{\mu}\right]=\sum_{\vec{x}, \mu} \frac{1}{2}\left[w_{i}^{\dagger}(x+\hat{\mu})\left(1+\gamma_{\mu}\right) U_{\mu}^{\dagger}(x) A_{\mu}(x) v_{j}(x)-w_{i}^{\dagger}(x)\left(1-\gamma_{\mu}\right) U_{\mu}(x) A_{\mu}(x) v_{j}(x+\hat{\mu})\right]$.

and allows us to construct the diagrams in Fig. 2 in terms of traces over products of meson fields. This method for dealing with conserved currents in the all-to-all set-up has also been understood for the DWF and overlap cases.

In practice we generate sets of meson fields for different $\gamma$-structures and with and without photon fields on a super computer. The contraction of meson fields to form correlation functions can be done off-line on a single node, increasing flexibility.

A further meson field is required for the lepton coupling diagram in Figure 2a. We place the leptonic part $L=\Gamma_{W}^{\mu} D_{\text {lepton }}^{-1} V_{\mu}^{c} A_{\mu}$ with the left-handed V-A current $\Gamma_{W}^{\mu}=\gamma_{\mu}\left(1-\gamma_{5}\right)$ in the meson field for the decay operator. 


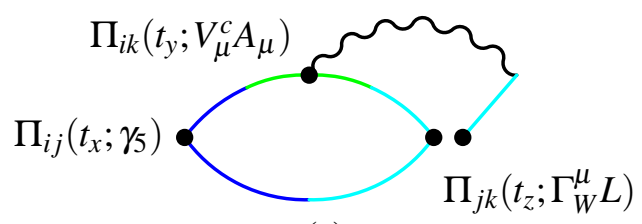

(a)

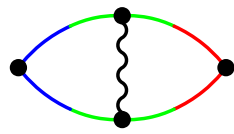

(b)

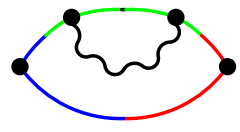

(c)

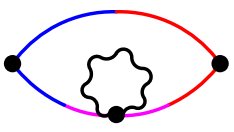

(d)

Figure 5: The diagrams shown here correspond to the QED correction to the decay rate. Here the diagrams are coloured corresponding to the type of meson field used to construct the correlator.

\subsection{Leptonic decay corrections from meson fields}

Using the meson fields discussed above it is possible to construct all the diagrams required for a calculation of corrections to the decay rate. This is illustrated in Figures 5a-5d where the colours correspond to the different meson fields required to construct each graph. In particular, blue for $\gamma_{5}$, red for $\gamma_{0} \gamma_{5}$, green for the conserved vector current with a photon insertion, light blue for the weak Hamiltonian and lepton insertion and pink for the tadpole insertion. In total five meson fields are required to determine the QED IB corrections to the decay rate.

The quark-disconnected diagrams (Figure 6) can be formed from the same set of meson fields without any further inversions.

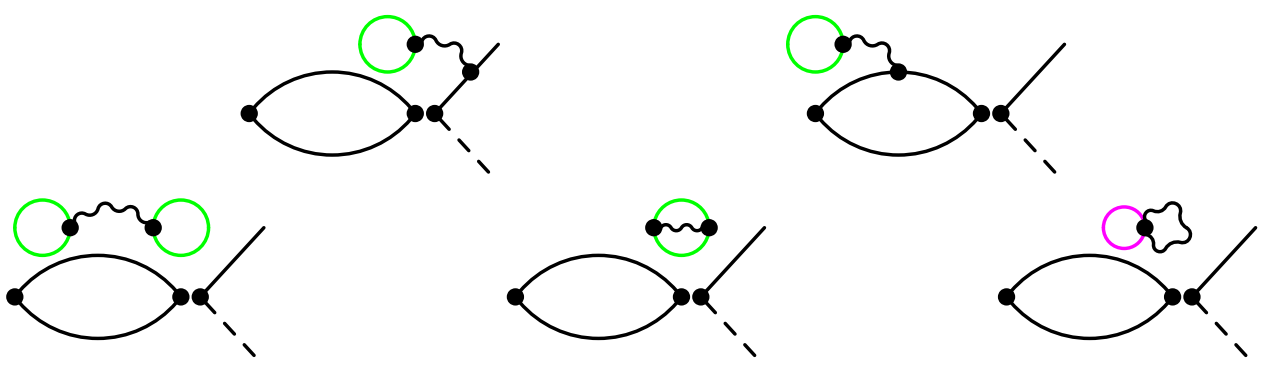

Figure 6: The set of disconnected diagrams that contribute to the QED correction to a leptonic decay at order $\alpha$. The colour coding corresponds to the meson fields that are used to construct the disconnected part.

\section{Conclusion}

Progress is being made towards a determination of the isospin breaking corrections to leptonic decays of pions and kaons. Here we presented one way of organising the workflow, namely in terms of meson fields, which offer a convenient approach for computing $n$-point functions from simple building blocks off-line. Apart from a few technicalities the above discussion carries over to Domain Wall Fermions, which we use in our core simulation program. We are in the process of testing the all-to-all approach and implementation of meson-field generation. Once we verify the all-to-all method for the calculation of QED effects we aim to calculate IB correction to leptonic decays for both the pion and kaon in this way. If this approach is successful a number of physics processes can be calculated from a set of stored meson fields, increasing the physics output from consumed computer time. 


\section{Acknowledgements}

A.P. and V.G. are funded in part by the European Research Council (ERC) under the European Union's Horizon 2020 research and innovation programme under grant agreement No 757646 and UK STFC grant ST/P000630/1. F.Ó.h. is funded by a scholarship from the Scottish Funding Council. A.J. received funding from STFC consolidated grant ST/P000711/1 and from the European Research Council under the European Union's Seventh Framework Program (FP7/2007-2013) / ERC Grant agreement 279757. C.T.S is partially supported by an Emeritus Fellowship from the Leverhulme Trust. J.R acknowledges support from STFC for his studentship. This work used the DiRAC Extreme Scaling service at the University of Edinburgh, operated by the Edinburgh Parallel Computing Centre on behalf of the STFC DiRAC HPC Facility (www.dirac.ac.uk). This equipment was funded by BEIS capital funding via STFC capital grant ST/R00238X/1 and STFC DiRAC Operations grant ST/R001006/1. DiRAC is part of the National e-Infrastructure. The authors acknowledge the use of the IRIDIS High Performance Computing Facility in the completion of this work.

\section{References}

[1] N. Carrasco, V. Lubicz, G. Martinelli, C. T. Sachrajda, N. Tantalo, C. Tarantino, and M. Testa. Qed corrections to hadronic processes in lattice qcd. Phys. Rev. D, 91:074506, Apr 2015.

[2] J. Foley, K. Jimmy Juge, A. O’Cais, M. Peardon, S. M. Ryan and J. I. Skullerud, Comput. Phys. Commun. 172 (2005) 145 doi:10.1016/j.cpc.2005.06.008 [hep-lat/0505023].

[3] S. Aoki et al Review of lattice results concerning low-energy particle physics. The European Physical Journal C, 77(2):112, Feb 2017.

[4] M. Hayakawa and S. Uno, Prog. Theor. Phys. 120 (2008) 413 doi:10.1143/PTP.120.413 [arXiv:0804.2044 [hep-ph]].

[5] S. Borsanyi et al., Science 347 (2015) 1452 doi:10.1126/science.1257050 [arXiv:1406.4088 [hep-lat]].

[6] T. Blum, R. Zhou, T. Doi, M. Hayakawa, T. Izubuchi, S. Uno and N. Yamada, Phys. Rev. D 82 (2010) 094508 doi:10.1103/PhysRevD.82.094508 [arXiv:1006.1311 [hep-lat]].

[7] Z. Davoudi, J. Harrison, A. Jüttner, A. Portelli and M. J. Savage, arXiv:1810.05923 [hep-lat].

[8] P. Boyle, V. Gülpers, J. Harrison, A. Jüttner, C. Lehner, A. Portelli, and C.T. Sachrajda. Isospin breaking corrections to meson masses and the hadronic vacuum polarization: a comparative study. Journal of High Energy Physics, 2017(9):153, Sep 2017.

[9] F. Bloch and A. Nordsieck, Phys. Rev. 52 (1937) 54. doi:10.1103/PhysRev.52.54

[10] G. M. de Divitiis et al. [RM123 Collaboration], Phys. Rev. D 87 (2013) no.11, 114505 doi:10.1103/PhysRevD.87.114505 [arXiv:1303.4896 [hep-lat]].

[11] D. Giusti, V. Lubicz, C. Tarantino, G. Martinelli, S. Sanfilippo, S. Simula and N. Tantalo, Phys. Rev. D 95 (2017) no.11, 114504 doi:10.1103/PhysRevD.95.114504 [arXiv:1704.06561 [hep-lat]].

[12] P. A. Boyle, A. Jüttner, C. Kelly and R. D. Kenway, JHEP 0808 (2008) 086 doi:10.1088/1126-6708/2008/08/086 [arXiv:0804.1501 [hep-lat]].

[13] C. Allton et al. [RBC-UKQCD Collaboration], Phys. Rev. D 78 (2008) 114509 doi:10.1103/PhysRevD.78.114509 [arXiv:0804.0473 [hep-lat]].

[14] C. Lanczos, Journal of Research of the National Bureau of Standards Vol. 45, No.4, October 1950, Research Paper 2133 